\title{
Efficient Bypass in Mesh and Torus NoCs
}

\author{
Iván Pérez*, Enrique Vallejo* and Ramón Beivide* \\ *University of Cantabria \\ Santander, Spain
}

\begin{abstract}
Minimizing latency and power are key goals in the design of NoC routers. Different proposals combine lookahead routing and router bypass to skip the arbitration and buffering, reducing router delay. However, the conditions to use them requires completely empty buffers in the intermediate routers. This restricts the amount of flits that use the bypass pipeline especially at medium and high loads, increasing latency and power.

This paper presents $N E B B$, Non-Empty Buffer Bypass, a mechanism that allows to bypass flits even if the buffers to bypass are not empty. The mechanism applies to wormhole and virtual-cut-through, each of them with different advantages. $N E B B$-Hybrid is proposed to employ the best flow control in each situation. The mechanism is extended to torus topologies, using FBFC and shared buffers.

The proposals have been evaluated using Booksim, showing up to $75 \%$ reduction of the buffered flits for single-flit packets, which translates into latency and dynamic power reductions of up to $30 \%$ and $23 \%$ respectively. For bimodal traffic, these improvements are 20 and $21 \%$ respectively. Additionally, the bypass utilization is largely independent of the number of $\mathrm{VCs}$ when using shared buffers and very competitive with few private ones, allowing to simplify the allocation mechanisms.
\end{abstract}

Index Terms-NoC, bypass router, hybrid flow control

\section{INTRODUCTION}

NoC latency has a clear impact on memory access time, and thus on the system performance. One of its most critical components is the router delay, because of the traditional pipelined implementation with several stages.

To minimize such latency, different mechanisms have been proposed to reduce the router pipeline stages, including lookahead routing [1] and router bypass [2]. Together, these mechanisms allow for a single-cycle router implementation, plus one cycle for link traversal. Additionally, bypass mechanisms reduce the use of buffers, which are the most power hungry components in the router. For these reasons, maximizing the utilization of the bypass pipeline is important for NoC latency and power reduction.

The bypass path is used when certain conditions (the bypass conditions) hold, as detailed in Section II-B. The bypass conditions used in previous proposals [2][3] guarantee that packets do not interleave in the same buffer (corrupting data) and, by construction, also preserve order for packets sent in the same path and virtual channel (VC), even when they do not belong to the same flow. Message ordering is not a

This work was supported by the Spanish Ministry of Science, Innovation and Universities, FPI grant BES-2017-079971, the Spanish Ministry of Science, Innovation and Universities under contract TIN2016-76635-C2-2-R (AEI/FEDER, UE) and the European HiPEAC Network of Excellence. The Mont-Blanc project has received funding from the European Union's Horizon 2020 research and innovation programme under grant agreement No 671697. requirement of many coherence protocols (or, at least, not in all the virtual networks) and it is in fact not guaranteed with many other mechanisms such as adaptive routing [4], [5], deflective routing [6], [7], [8] or dynamic $\mathrm{VC}$ assignment [9]. Additionally, it is unnecessarily restrictive for bypass conditions, since it prevents bypass in cases in which it could be used.

The current paper reviews and extends the work originally announced in [10]. This research proposes Non-Empty Buffer Bypass (NEBB), a novel approach for the use of the bypass that increases its utilization and effectiveness. With $N E B B$, bypassed packets can overtake buffered packets. Our proposal can be implemented under different flow control mechanisms, and depending on the buffer occupancy, it is more efficient to employ either Wormhole (WH) or Virtual Cut-Through (VCT). Based on this observation, we design NEBB-Hybrid, a mechanism that dynamically selects between WH or VCT forwarding in the bypass path, maximizing the amount of packets that use this shortcut. The mechanisms have been implemented using private or shared buffers in mesh and torus topologies. In the later using Flit-Bubble Flow Control (FBFC [11]) to efficiently avoid routing deadlock, given that NEBB does not require empty buffers to forward packets as required by other bypass router architectures [2], [3], [12]. This paper extends and completes our previous seminal work with the following contributions:

- An extension of the evaluations of [10] with a comparison between the flow control used in the original bypass routers in [2], [3], [12], which requires empty buffers to forward packets, and the traditional WH mechanism used as our baseline.

- An implementation of NEBB in torus, using Flit-Bubble Flow Control (FBFC) [11] with shared buffers.

- A detailed evaluation in mesh and torus NoCs, which shows reductions up to $75 \%$ in buffer utilization that translate into latency and power reductions up to $30 \%$ and $23 \%$ respectively.

- An evaluation of NEBB under real-traffic workloads, using gem5 [3] simulations driven by the PARSEC suite benchmarks [13].

- An analysis of potential starvation problems when giving priority to the bypass path over the standard path, which concludes that starvation is not an issue under realistic conditions.

The organization of the paper is as follows: Section II introduces lookahead bypass routers and flow control mechanisms; Section III describes the $N E B B$ and Hybrid proposals, including the extension to torus networks with FBFC; Section IV and V detail the methodology and present the results; Section VI 


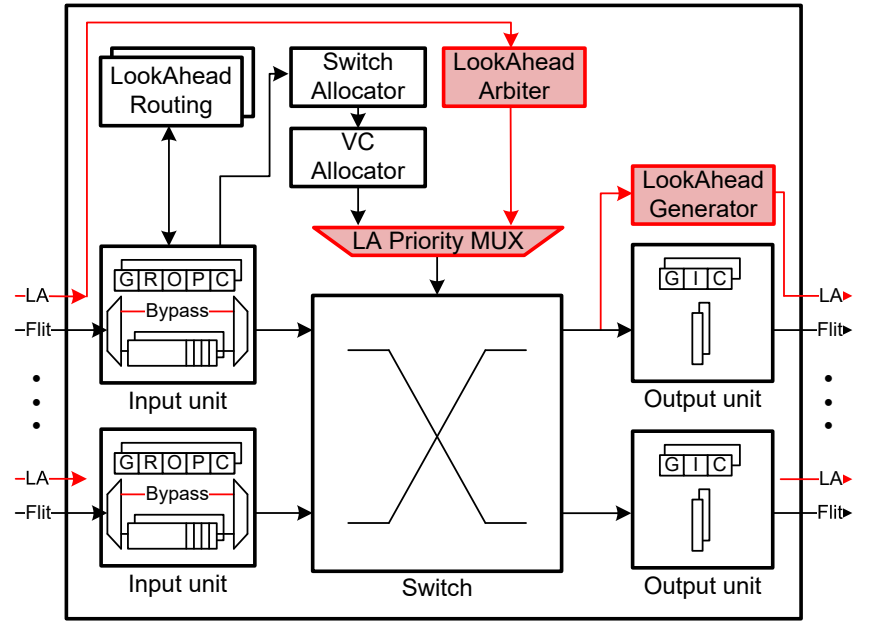

Fig. 1: Lookahead (LA) bypass router architecture.

discusses relevant related work; and Section VII concludes the work.

\section{BACKGROUND}

This section presents the required background on LookAhead bypass routers and on the use of WH or VCT in the NoC.

\section{A. LookAhead Bypass Router Architecture}

This section describes a LookAhead bypass router architecture. Lookahead bypass routers short-cut the buffer write and arbitration pipeline stages in the absence of flit conflicts; otherwise, the traditional (non-bypass) pipeline is used. The implementation relies on control packets, denoted advance bundles, or LookAheads (LA), which setup the bypass one cycle before the arrival of a flit. LAs are generated after flits win access to the crossbar. They are destroyed in the next router, after configuring the path or because of conflicts.

Figure 1 depicts the router architecture based on the proposal of Krishna et al. [12]. The architecture extends a traditional router with some additional units to support the bypass forwarding (shaded in the figure): LookAhead Arbiter, LA/flit Priority and LookAhead Generator. The LookAhead Arbiter is optional as discussed in Section II-B; if not implemented, a LookAhead Conflict Check unit is required, which removes all conflicting LAs. The LA/flit Priority unit gives absolute priority to the LAs over the buffered flits, or vice versa.

Figure 2 represents the two pipelines of the router, used in two consecutive routers R0 (non-bypass pipeline) and R1 (bypass pipeline). White boxes represent the pipeline stages for flits (data) and dark red boxes the stages of the LAs used to pre-setup the crossbar. The functionality of each stage in both pipelines are described next.

1) Pipeline functions associated to flits:

a) Buffer Write (BW) and LA Routing (LA-R): Flits are received and stored in the requested $\mathrm{VC}$. When a head flit reaches the front of a buffer, the LA route (for the next hop) is computed.

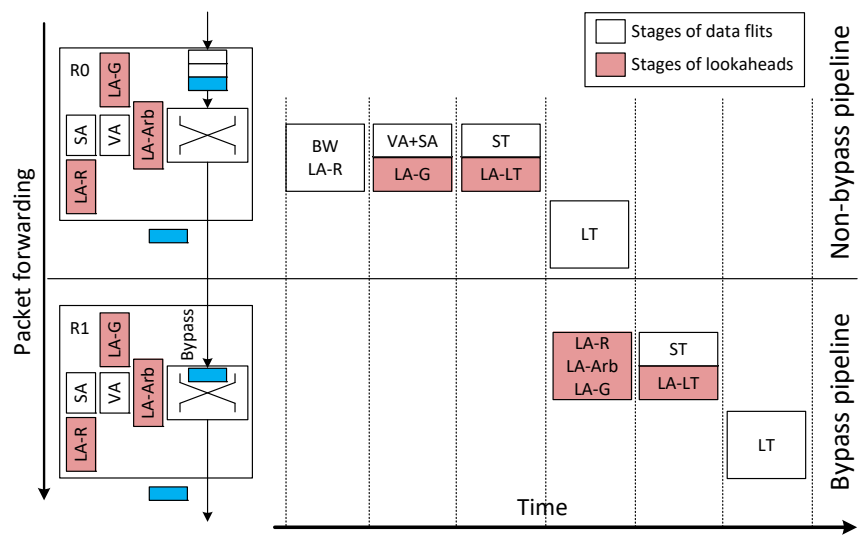

Fig. 2: Lookahead bypass router pipelines. Acronyms are defined in the text in Section II-A.

b) Virtual Channel Allocation (VA): A head flit places a request for a destination $\mathrm{VC}$ into the $\mathrm{VC}$ allocator. The VC allocator chooses winners for each output among all input VCs. These allocations remain until a tail flit is forwarded.

c) Switch Allocation (SA): Flits are granted the use of the crossbar. The switch allocator arbitrates among the output requests received from the input ports in this stage.

d) Switch Traversal (ST) and Link Traversal (LT): winners of SA traverse the router crossbar to the output port, and then the link to the next router or destination node. When a LA wins access to the crossbar, the associated flit skips BW, VA and SA and goes to the ST and LT stages.

The distribution of functions into stages may vary; Section IV presents the implementation used in our evaluations.

2) Pipeline functions associated to lookaheads:

a) LookAhead Routing (LA-R): it computes the packet route one hop in advance, using the information from the received LA.

b) LookAhead Arbiter (LA-Arb): LAs must reserve the desired output port in order to set the bypass for the upcoming flit in the next cycle. This functions handles conflicts between different LAs requesting the same output port. Different implementations are considered: either no LA can proceed in case of conflict (function LA-Conflict Check, no arbiter) or an arbiter per output port is used to select one winner (function LA-Arb as depicted in Figure 1).

In addition, buffered flits in SA/VA stages can conflict with winning lookaheads. In case of such conflicts, priority is given to either buffered flits [2] or LAs [3], [12].

c) LookAhead Generation (LA-G) and Link Traversal (LA$L T)$ : it creates the control information for a flit and sends it during its ST stage. In this way, LAs arrive to the next router one cycle before their flits.

\section{B. Lookahead Bypass Router Policies}

In a Lookahead bypass router, the bypass path is used only if the following bypass conditions are met:

1) The buffer at the input port that receives the LA is empty.

2) There is no output port conflict with buffered flits. 
3) There are no conflicts between LAs arriving in the same cycle.

Condition 1 guarantees that packets do not interleave in the same buffer (as discussed in section III-A) and are forwarded in order. With multiple virtual channels, this restriction applies to the buffer of the $\mathrm{VC}$ where the flit would be stored in case of using the non-bypass pipeline. Many proposals employ a large number of VCs [2], [3] to avoid limitations in the bypass from this condition. However, this requires a large buffer area and complicates the $\mathrm{VC}$ allocator, which typically sets the critical path delay of the router [14].

Condition 2 gives absolute priority to flits in the non-bypass pipeline, this is, those already stored in the pipeline buffers. Note that the opposite priority may also be considered to maximize the utilization of the bypass path. The impact of the selected priority is evaluated in Section V-F2.

Condition 3 implies that there is no arbiter between LAs: when multiple LAs contend for the same output, they are all discarded and the associated flits use the non-bypass pipeline.

Different implementations may modify slightly these conditions. In [3], [12] absolute priority is given to LAs over packets in the buffers (condition 2 is removed); and an arbiter per output port is implemented to select one wining LA in case of conflict for an output port (condition 3).

\section{Flow Control and deadlock avoidance}

Flow control in NoCs is applied at flit level when using WH. WH forwards traffic on a flit-by-flit basis, based on the availability of space for each flit in the buffer of the next router. WH allows to reduce buffer size, since routers do not need to accommodate a complete packet.

However, to avoid unnecessary throttling buffers need to be sized for the buffer turnaround time, this is, the minimum idle time required for successive flits to reuse a buffer. A typical turnaround time is 4 to 6 cycles [14], [15], similar to typical NoC packet sizes in flits. Such amount of buffering allows to implement Virtual-Cut Through (VCT) flow control, which forwards data on a packet-by-packet basis.

Multiple NoC designs rely on an additional restriction to forward data, requiring a free and empty $\mathrm{VC}$ in the next selected router to forward a packet. This simplifies the control, reducing the amount of signals sent between adjacent routers, and has been implemented in previous work such as [2], [3], [12] Requiring the emptiness of the destination $\mathrm{VC}$ is a conservative solution to guarantee the first bypass condition mentioned in Section II-B. A packet that wins SA and starts to progress to the next router can only happen if the next $\mathrm{VC}$ is empty, hence LAs always find that the input buffer in the router to bypass is empty. These proposals follow a flit level switch allocation, so the employed flow control can be considered as a restricted implementation of WH. From now on, we refer to this flow control as WH - Empty $V C$ or simply Empty $V C$.

Our proposal uses WH or VCT regulated by credits, without the previous emptiness requirement. This requires additional logic to check the first condition of the bypass. Every LA has to check, in the router to bypass, that the input buffer is empty and, in the case of $\mathrm{WH}$, inactive as well. This is because in

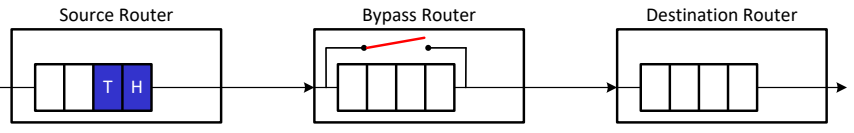

(a) Initial buffer occupation to bypass the blue packet applying Empty $V C$.

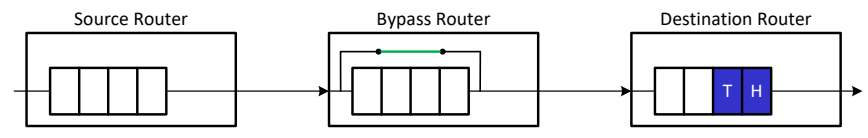

(b) Bypass of the blue packet applying Empty $V C$.

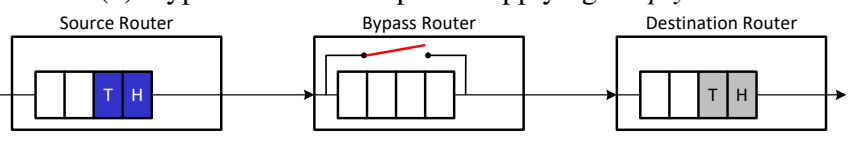

(c) Initial buffer occupation to bypass the blue packet applying $\mathrm{WH}$ or VCT.

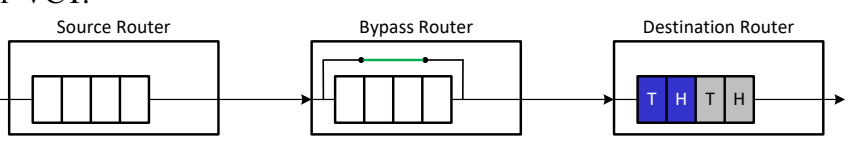

(d) Bypass of the blue packet applying WH or VCT.

Fig. 3: Example of packet bypassing applying Empty $V C$ and WH (VCT is equivalent in this example). Empty $V C$ requires both buffers empty, in the bypass router and in the destination router, to advance. WH or VCT requires space for a flit or packet, respectively to advance. Taking the bypass requires the emptiness of the bypass router buffer and room for a flit (WH) or a packet (VCT) in the destination router.

WH there can be holes (time gaps) between consecutive flits of a packet. Therefore, LAs can see the buffer empty but active, i.e. being used by another packet whose tail flit has not been sent yet.

Figure 3 depicts an example bypassing a packet (represented in blue), applying either Empty VC (Figures $3 \mathrm{a}$ and $3 \mathrm{~b}$ ) or WH (Figures $3 \mathrm{c}$ and $3 \mathrm{~d}$ ). There are three routers: source, bypass and destination ${ }^{1}$, with packets stored in their buffers. Empty $V C$ requires the emptiness of the buffers in the bypass and destination routers. Nevertheless, our router implementations equipped with bypassing only requires the emptiness of the bypass router buffer and room for a flit or a packet in the destination router depending if the flow control applied is $\mathrm{WH}$ or VCT.

Many NoC designs employ link widths that accommodate a whole packet [16], [17], [18]. In such case, packets are singleflit and there is no difference between WH and VCT. Other designs present bimodal traffic, with 1 and 5 flits being typical values [11]. For such cases, WH credit accounting is performed for each flit of the packet, whereas in VCT it is done for the whole packet. This accounting has to be carefully considered when both mechanisms are mixed, particularly when using shared buffers.

Deadlock avoidance in a mesh is often implemented using routing restrictions, such as Dimension-Ordered Routing (DOR). In torus networks additional restrictions are necessary. For example, Dateline [19] breaks the cyclic dependency chain of the physical links using at least 2 VCs. The packets in a

\footnotetext{
${ }^{1}$ They do not necessarily refer to the source and destination of the packet.
} 


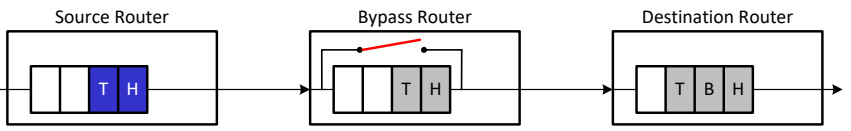

(a) Initial state. $H, B$ and $T$ denote head, body and tail flit. Source Router

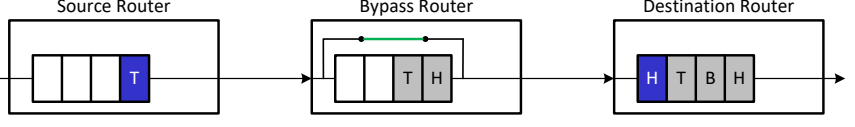

(b) Incorrect bypass of the blue head flit.

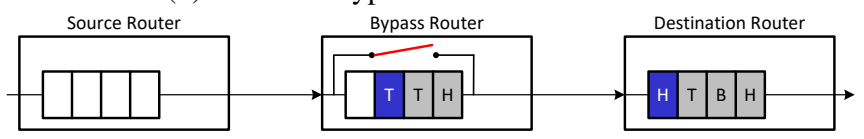

(c) Blue tail flit is buffered at the bypass router because there is no room at the destination router, causing packet interleaving in the same buffer and data corruption.

Fig. 4: Incorrect packet bypass, which interleaves data in the same buffers.

ring initially only use one of the two VCs and the second $\mathrm{VC}$ is reserved for those packets that cross the Dateline, i.e. a predefined router in the ring. A more efficient solution is Flit-Bubble Flow Control (FBFC) [11], a derivative mechanism of Bubble Routing [20] for WH. These mechanisms leverage the flow control to avoid deadlock in the network. FBFC adds an injection rule to avoid deadlock: a packet is injected only when there is space for the whole packet plus one flit, i.e. the bubble. This rule guarantees the progress of flits that are already in the ring. The same applies to packets that change dimension.

\section{EFFicient Bypass in Mesh AND TORUS}

This section first introduces our Non-Empty Buffer Bypass proposal, detailing VCT and WH implementations. Then, it introduces the Hybrid mechanism which combines VCT and WH to maximize bypass. Finally, some implementation details are presented.

\section{A. Non-Empty Buffer Bypass}

Non-Empty Buffer Bypass (NEBB) performs bypass when the router buffer is not empty, as long as this does not interleave flits from different packets in a single virtual channel. NEBB relaxes the bypass condition 1 introduced in Section II-B to the following two general conditions, which depend on the flow control mechanism:

1a) No packet in the bypassed input VC is already advancing to an output port.

1b) The packet may be forwarded without packet interleaving in a buffer, according to the flow control employed.

The VC control registers (GROPC in Figure 1) are updated for both bypass and ordinary packet forwarding. Condition 1a is required to avoid that bypassed packets conflict with other packets that have already won allocation and have their status recorded in these $\mathrm{VC}$ control registers.

Condition $1 \mathrm{~b}$ is required to prevent data corruption, and it is dependent on the flow control used. Figure 4 shows an example of incorrect packet bypass using $\mathrm{WH}$, to illustrate this requirement. The dark blue packet in the source router in Figure $4 \mathrm{a}$ tries to bypass the intermediate router. The Head $(\mathrm{H})$ flit is bypassed in Figure 4b because there is a free buffer in the destination router buffer (following $\mathrm{WH}$ ) and overtakes the packet in the non-empty buffer of the bypass router. The next flit (tail, T) cannot be bypassed because there is no more room in the destination router buffer, so it is stored in the buffer of the bypass router in Figure 4c, behind the existing packet. In this situation, the grey packet is interleaved in the same VC with the blue packet, so data is corrupted. Even if the grey packet is forwarded to a different output, the blue tail flit has lost its routing and status information from the VC registers, so it cannot be forwarded.

Table I summarizes the different cases in which bypass can be applied for each flow control mechanism, with and without $N E B B$. Note that in the base mechanisms without $N E B B$ both implementations of $\mathrm{WH}$, with and without the Empty $V C$ requirement, present the same behavior because the requirement of the empty buffer is already included in the original bypass conditions. The specific forwarding conditions for $N E B B$ using WH or VCT are presented next. These conditions rely on the occupancy level of the buffers in the bypass and destination routers.

1) $N E B B-W H$ : Under wormhole, arbitration is performed flit by flit. Therefore, the bypass of one flit does not guarantee that the following flits of the packet will be also bypassed. Figure 4 has already illustrated an incorrect case of using WH with NEBB. For this reason, multi-flit packet bypass is unsafe when the bypass router buffer is not empty.

The solution for NEBB-WH is to avoid bypassing multi-flit packets when the bypass router buffer is not empty. By contrast, NEBB-WH can bypass single-flits packets, which is a frequent case. Therefore, condition 1 under WH flow control results as follows:

1a) No packet in the input buffer (VC) is already advancing to an output port.

1b) The packet is single-flit or the bypass buffer is empty.

2) NEBB-VCT: Under Virtual Cut-Through, arbitration is performed once per packet and the assigned resources remain allocated for the duration of the packet forwarding. This prevents any packet interleaving, avoiding the problem presented in Figure 4.

However, to forward a packet VCT requires space for the whole packet at the destination buffer. This means that in NEBB-VCT multi-flit packets cannot be bypassed if the destination router buffer can only accommodate part of the packet. Additionally, the buffer in the bypass router also needs room to accommodate the whole packet, even if it is not used: otherwise, the source router would not start sending the packet. Therefore, condition 1 under VCT results as follows:

1a) No packet in the input buffer (VC) is already advancing to an output port.

1b) The bypass and destination buffers have room for the whole packet.

Figure $5 b$ illustrates the bypass of packets in VCT. As there is room for the whole packet in the bypass and destination routers, the blue packet can be bypassed, independently of the 
TABLE I: Bypass buffer conditions for different mechanisms. bypass buffer refers to the buffer in the bypass router being empty or not. Dest. buffer indicates if the destination buffer may accommodate the whole packet or only some flits (partial)

\begin{tabular}{|c|c|c|c|c|c|c|}
\hline \multirow{3}{*}{$\begin{array}{l}\text { Bypass type } \\
\text { (Required buffer size) }\end{array}$} & \multicolumn{3}{|c|}{ Bypass buffer: empty } & \multicolumn{3}{|c|}{ Bypass buffer: not empty } \\
\hline & \multirow[t]{2}{*}{ Single-flit packet } & \multicolumn{2}{|c|}{$\begin{array}{ll}\text { Multi-flit packet } \\
\end{array}$} & \multirow[t]{2}{*}{ Single-flit packet } & \multicolumn{2}{|c|}{$\begin{array}{ll}\text { Multi-flit packet } \\
\end{array}$} \\
\hline & & Dest. buffer: packet & Dest. buffer: partial & & Dest. buffer: packet & Dest. buffer: partial \\
\hline VCT (packet size) & $\checkmark$ & $\checkmark$ & $\mathrm{X}$ & $\mathrm{X}$ & $\mathrm{X}$ & $\mathrm{X}$ \\
\hline WH - Empty VC ${ }^{2}$ (1 flit) & $\checkmark$ & $\sqrt{ }$ & $\checkmark$ & $\mathrm{X}$ & $\mathrm{X}$ & $\mathrm{X}$ \\
\hline WH - Baseline (1 flit) & $\checkmark$ & $\checkmark$ & $\checkmark$ & $\mathrm{X}$ & $\mathrm{X}$ & $\bar{X}$ \\
\hline NEBB-WH (1 flit) & $\sqrt{ }$ & $\checkmark$ & $\sqrt{ }$ & $\checkmark$ & $\bar{X}$ & $\bar{X}$ \\
\hline NEBB-VCT (packet size) & $\checkmark$ & $\checkmark$ & $\mathrm{X}$ & $\checkmark$ & $\sqrt{ }$ & $\mathrm{X}$ \\
\hline NEBB-Hybrid $\left(1\right.$ flit $\left.^{3}\right)$ & $\checkmark$ & $\checkmark$ & $\checkmark$ & $\bar{\checkmark}$ & $\checkmark$ & $\mathrm{X}$ \\
\hline
\end{tabular}

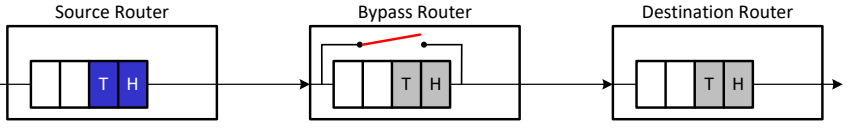

(a) Initial state of the example.

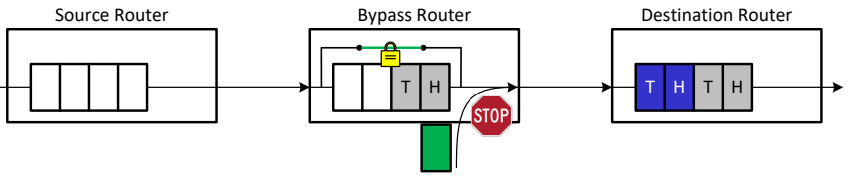

(b) Bypass allowed as there is room for the blue packet in the destination.

Fig. 5: NEBB VCT bypass. A packet is bypassed to the destination router, with room for the whole packet in the destination and a not empty buffer in the bypass router.

emptiness of the buffer in the bypass router. During packet bypass the resources are reserved (locked), so other buffered flits or lookaheads (e.g. the green flit in a different port) cannot obtain the output port that is using the blue packet, until the tail flit of the blue packet reaches the ST stage of the bypass router.

\section{B. Improved bypass in NEBB via Hybrid Flow Control}

The NEBB-Hybrid mechanism (or simply Hybrid) dynamically selects between WH and VCT to maximize the utilization of the bypass. Section III-A and Table I present the limitations of $N E B B$ when using each mechanism: $N E B B-W H$ does not bypass multi-flit packets when the bypass router buffer is not empty; NEBB-VCT does not bypass multi-flit packets when the bypass router buffer cannot accommodate the whole packet. Hybrid selects the most suitable mechanism in each case.

In Hybrid the standard pipeline uses WH. The bypass pipeline uses both flow controls: if the buffer to bypass is empty, the router checks if there is room for a flit in the destination $\mathrm{VC}$, following $\mathrm{WH}$; otherwise, the router checks if there is room for the whole packet, following VCT. For single-flit packets, both mechanisms are equivalent.

Combining two different flow control mechanisms introduces subtle implementation requirements. The Hybrid system employs WH, so packets from different VCs may be interleaved in the same physical channel. In such case, a "hole" appears in the forwarding of the flits of a packet. Allocation in Hybrid is implemented flit by flit, to take advantage of such holes and maximize forwarding.

However, the original NEBB-VCT forwarding for multi-flit packets is safe because it guarantees that the whole packet is forwarded consecutively, as presented in Section III-A2. To preserve this safeguard with flit by flit allocation and VCT, Hybrid employs variable priority arbiters in the LA Arbiter. Maximum priority is assigned to the LAs of multi-flit packets bypassed by VCT. For each output port, there can be at most one packet with maximum priority. The holes of this packet are leveraged to bypass flits from other packets, but only following WH (including single-flit packets), to avoid having to assign maximum priority to two packets in the same output port.

\section{Credit management using shared buffers}

Shared buffers [21], [9] improve efficiency. Shared buffer capacity accounting needs to consider the dual flow control. Packets advancing following VCT have to reserve room for their size in advance. Otherwise, other packet advancing to another $\mathrm{VC}$ of the same input (using WH) may invade slots initially intended for the first packet.

The evaluations of this work use credits, so the reservation is done decrementing the credit count by the packet size when bypassing a packet via VCT, or flit by flit via WH.

\section{VC management: hardware considerations}

The router architectures proposed in [2], [12] combine a pool of free VCs together with on/off signaling and a VC Selector (VS), instead of traditional Virtual channel Allocation (VA), to simplify the VC management and, therefore, reduce the length of the input path that may determine the critical path. The on/off signalings or free $V C$ signals, replace credits by indicating to the upstream router whether the current router has a free VC in the pool or not. VS selects a free VC when a packet arrives to the router instead of assigning the $\mathrm{VC}$ in the upstream router after a packet wins VA. The proposed router architecture for the different versions of NEBB uses traditional credits and VA, but it is compatible with on/off signaling and VS. Instead of using free $V C$ signals, NEBB can use avail VCs signals to indicate if there is an available VC with enough available slots to store a whole packet. In the case of NEBB-VCT and NEBB-Hybrid, they require one avail VC signal per packet size, in order to perform VCT forwarding when bypassing the whole packet.

Another implication of implementing NEBB is the extra logic necessary to check the additional bypass conditions. We assume that the overhead incurred is low because the conditions

\footnotetext{
${ }^{2}$ Requires the emptiness of the destination buffer.

${ }^{3}$ Packets larger than the buffer size cannot be bypassed by VCT rules.
} 
are simple and they can be pre-evaluated in the previous cycle. Besides, NEBB does not require VCs to achieve a high bypass utilization and throughput (see Section V) so the complexity of the $\mathrm{VC}$ management can be reduced drastically if required to meet timing requirements.

\section{E. NEBB in torus using Flit Bubble Flow Control}

Tori are considered good alternatives to meshes [22], [23] because they are node-symmetric, have lower diameter and double the bisection bandwidth of meshes due to their wraparound links. Like 2D-meshes, 2D-tori adapt well to the tiled organization of CMPs. However, wraparound links introduce routing cyclic dependencies.

This subsection presents the implementation details required to adapt $N E B B$ to a torus topology. In particular, the proposed design supports a torus using FBFC for deadlock avoidance and shared buffers, which is an efficient implementation. FBFC is introduced first. It is followed by a discussion on the implementation details of the allocation mechanisms, which may cause deadlock in torus topologies, and the specific details to avoid it when using $N E B B$.

Flit Bubble Flow Control (FBFC) [11] is an efficient deadlock avoidance mechanism for torus-like topologies. FBFC$\mathrm{L}$ (Localized) requires that when a packet is injected or changes its dimension there must be space for the whole packet plus one flit. This guarantees that at least one flit slot is always empty in a dimension. Note that FBFC does not support the Empty $V C$ mechanism described in Section II-C. In Empty $V C$ only one packet can coexist in a VC. A flit from another packet cannot advance in the ring and occupy the bubble because there is another packet (the injected one) in the buffer.

The switch allocator often employs a two-stage implementation [3], [12], first an arbiter for the inputs and then an arbiter for each output. Simple round-robin (RR) arbiters are often used. RR input arbiters cycle through all available VCs, selecting one at a time consecutively. If one of the output VCs is not available (for example, there are no credits in the destination VC) it does not proceed, wasting one cycle. However, such implementation simplifies the router design, since output availability does not need to be propagated to the inputs. Also, such implementation inherently multiplexes packet flits, generating packet holes in WH.

Holes are undesirable when VCT is also employed in the bypass in Hybrid, as discussed in Section III-B. To minimize them, we use a variable priority input arbiter, selecting the same VC until the packet tail flit is forwarded, similar to VCT. However, this might introduce performance and deadlock issues when WH and VCT are combined: First, a packet may be forwarded by WH without space at the destination buffer for the complete packet, introducing delays until the buffer becomes available. Second, this introduces a dependence between the input VCs, which generates a deadlock when a delayed packet in a buffer waits for another buffer that is full and waiting for the local arbiter to finish forwarding a packet from a different $\mathrm{VC}$, also delayed. This deadlock problem is explained in more detail next with an example.

The configuration presented in Figure 6 has three routers forming a ring (1D-Torus), unidirectional in this case for

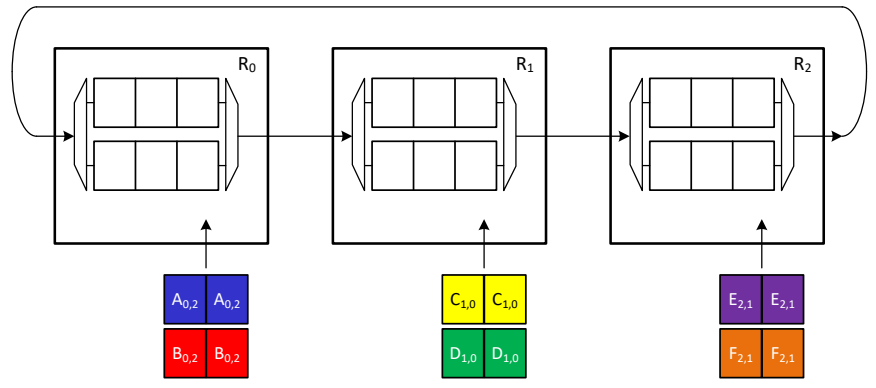

(a) Initially each router injects two packets.

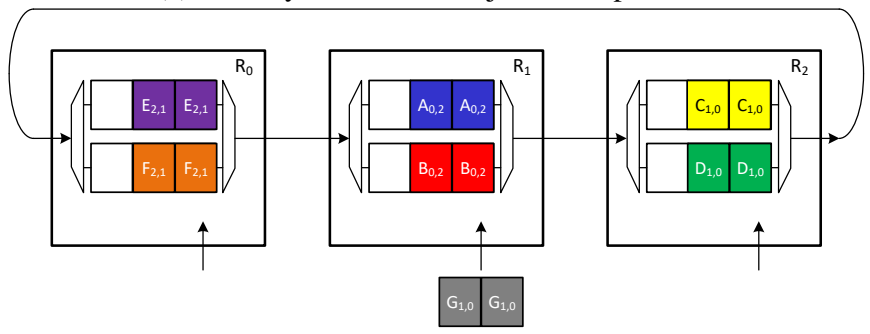

(b) $R_{1}$ injects packet $G$ after sending packet $D$ to $R_{0}$.

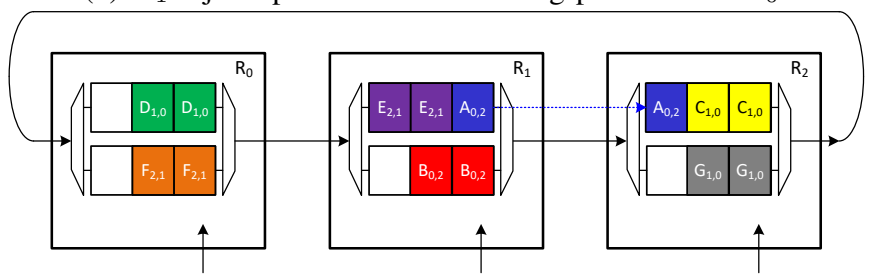

(c) SA-I winners: $E$ in $R_{0}, A$ in $R_{1}$ and $D$ in $R_{2}$.

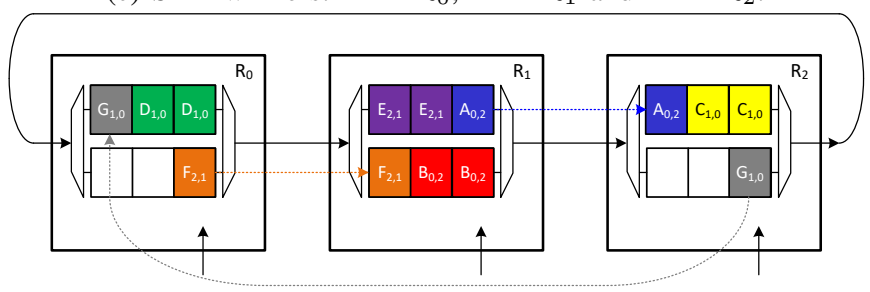

(d) $F$ wins in $R_{0}$ and $F$ in $R_{2}$ producing a deadlock.

Fig. 6: Switch allocator deadlock in Torus (ring) using FBFC. Packets have two flits. They are represented with different colours and letters $\left(X_{s r c, d s t}\right.$, where $X$ the packet identifier, $s r c$ the source router and $d s t$ the destination router).

simplicity. Every packet in the example has two flits and requires two hops to reach the destination router. Each packet is represented with a different colour and is identified by a letter, its source and destination: $X_{s r c, d s t}$, where $X$ is the identifier, $s r c$ the source and $d s t$ the destination.

Figure 6a depicts the initial state, where two packets are injected in each router: $A$ and $B$ in $R_{0} ; C$ and $D$ in $R_{1}$; and $E$ and $F$ in $R_{2}$. The FBFCL condition is satisfied for every packet because, at least, there is a VC in the next router with room for 3 flits (2 flits plus a bubble).

Figure $6 \mathrm{~b}$ represents the state of the routers after injecting these packets in the ring. In this situation there is a conflict between VCs in SA-I of each router. Assume that the winners are: $E$ in $R_{0}, A$ in $R_{1}$ and $D$ in $R_{2}$. And that they choose the first VC of $R_{1}, R_{2}$ and $R_{0}$, respectively. In addition, $R_{1}$ will inject a new packet, $G$, in the ring after $D$ frees the second $\mathrm{VC}$ 
TABLE II: Default simulation parameters.

\begin{tabular}{|c|c|}
\hline \multicolumn{2}{|r|}{ General parameters } \\
\hline Topology & $8 \times 8$ mesh or torus, concentration $c=4$ \\
\hline Link latency & 1 cycle \\
\hline Router architecture & 2/4-stage bypass router \\
\hline Router size & 8 ports ( 4 transit, 4 injection/ejection) \\
\hline Packet size & 1 and 5 flits \\
\hline Buffer implementation & Shared (DAMQ, [21]) \\
\hline Buffer size & 12 flits (1 private flit per VC) \\
\hline Routing & DOR \\
\hline SA input arbiters & 8 Round Robin arbiters, \#VCs:1 \\
\hline SA output arbiters & 8 Matrix arbiters, $8: 1$ \\
\hline LA arbiters & 8 Matrix arbiters, $8: 1$ \\
\hline VA policy & Highest number of credits \\
\hline Channel width & 128 bits \\
\hline \multicolumn{2}{|c|}{ Synthetic traffic parameters } \\
\hline Num. VCs & 2 \\
\hline Simulation cycles & 50.000 cycles \\
\hline \multicolumn{2}{|c|}{ Full-system parameters } \\
\hline CPUs & 64x O3 ARM @ 2 GHz (DerivO3CPU) \\
\hline L1 caches & $32 \mathrm{~KB}$ (L1-I) and 64KB (L1-D) per core \\
\hline L2 caches & $64 \times 256 \mathrm{~KB}$ shared banks \\
\hline Memory controllers & 16 (first and last mesh rows) \\
\hline Num. VCs & $3(1$ per VN) \\
\hline NoC frequency & $2 \mathrm{GHz}$ \\
\hline Simulation cycles & $10^{8}$ cycles \\
\hline \multicolumn{2}{|c|}{ DSENT parameters } \\
\hline Frequency & $2 \mathrm{GHz}$ \\
\hline Technology & Tri-Gate $11 \mathrm{~nm}$ LVT process \\
\hline
\end{tabular}

of $R_{2}$. The new state of the routers is depicted in Figure 6c. In this case $F$ wins in $R_{0}$ and $G$ in $R_{2} . G$ chooses the first VC of $R_{0}$ and $F$ the second VC of $R_{1}$. Finally, Figure $6 \mathrm{~d}$ shows the deadlock. In $R_{0}, F$ locks SA-I but cannot progress because the second VC of $R_{1}$ is full. The same occurs with $A$ in $R_{1} . B$ cannot advance to $R_{2}$ despite there is space the second VC because SA-I is locked by $A$. And in $R_{2}, G$ is blocked by the lack of space in the first VC of $R_{0}$, and $C$ cannot win SA-I because it is locked by $G$.

To avoid these issues, we give priority to body flits to minimize packet holes, but remove this priority when a flit does not advance, so the following ready $\mathrm{VC}$ is selected in the next arbitration cycle.

1) Bypass in torus using Flit Bubble Flow Control and shared buffers: The bypass mechanisms are compatible with a torus using FBFC and private buffers. However, shared buffers may introduce deadlock because the bubble condition is checked when the head of the packet is forwarded. Consider a shared buffer with space for exactly two packets. Two packets are forwarded, interleaved in the same physical channel, and they obey the condition when their head is sent. However, when they are fully received, the bubble disappears.

To avoid this issue, when multi-flit packets are forwarded to a shared buffer with a dimension change, the credit counter is decremented by the whole packet size when the head flit is sent, regardless of the flow control. Therefore, in $N E B B$ Hybrid credits are decremented by the whole packet in two cases: when multi-flit packets are forwarded using VCT and for injection and dimension change using any flow control.

\section{Methodology}

We have implemented the router architecture described in Section II and the bypass schemes described in Section III in BookSim [24]. We model a 256-core network, arranged as an $8 \times 8$ mesh or torus topology, with concentration $c=4$. The router employs a two-stage switch allocator similar to [3], [12] to balance pipeline stages. Priority is given to LAs over buffered flits. In the non-bypass pipeline, priority is given to body flits as presented in Section III-E. Simulation parameters are shown in Table II, unless otherwise noted in the text.

We employ six bypass models. Empty $V C+A r b$ implements Empty $V C$ using an arbiter between LAs. Baseline is a WH reference that uses LA conflict-check, while Baseline + Arb is a WH reference using an arbiter between LAs. Additionally, we implement the three $N E B B$ variants introduced in Section III: $N E B B-W H, N E B B-V C T$ (which also employs VCT in the nonbypass pipeline) and $N E B B-H y b r i d$.

Experiments use synthetic traffic, with single-flit packets or bimodal traffic. Bimodal traffic resembles a coherence protocol using packets of one (control) and five (data) flits. A singleflit packet ratio of $80 \%$ is used [11], [25]. The traffic pattern employed is random uniform, but we also evaluate bit-reversal, transpose and hotspot (with hotspots in nodes $0,15,240$ and 255). We measure relevant metrics such as average packet latency, dynamic power, and percentage of buffered flits. The latter divides the amount of times a flit is buffered by the total number of times a flits is forwarded, averaged for all flits.

We use gem5 [26] to evaluate NEBB-Hybrid with real traffic provided by Full-System (FS) simulations. We simulate a tiled system with 64 Out-Of-Order (O3) ARM CPUs with private L1s and a shared L2 distributed among the tiles. We use Virtual Networks (VN) to avoid protocol-deadlock. Each VN has one $\mathrm{VC}$, except for the case of for Empty $V C+A r b$ in the torus, which requires $2 \mathrm{VCs}$ to avoid the inherent routing-deadlock of rings when using Dateline [19]. We run the PARSEC suite [13] with the simlarge input set for every benchmark to have enough workload for the 64 cores. Because of the excessive simulation time of the whole benchmarks, we present results from the first 100 million cycles.

Dynamic power results are obtained using DSENT [27]. We have implemented a model of the bypass router based on the default four-stage router model of DSENT. The dynamic power of the buffers and allocators from DSENT is multiplied by the ratio of buffered flits over all the received flits per router. The LA arbiters employed are equal to the arbiters in the second stage of the switch allocator. Therefore, the LA arbiters power equals the power of the second stage of the switch allocator provided by DSENT. We use no correction factor because these arbiters are used for every LA, and one LA is received for each flit. We estimate that the extra control logic (such as the checks of the packet size, the occupancy of the buffer to bypass, etc) is negligible compared to the consumption of the buffers, crossbar or arbiters.

\section{RESUlts}

This section highlights the most notable empirical results when comparing the different router architectures. A performance comparison between the Empty $V C$ mechanism and our 


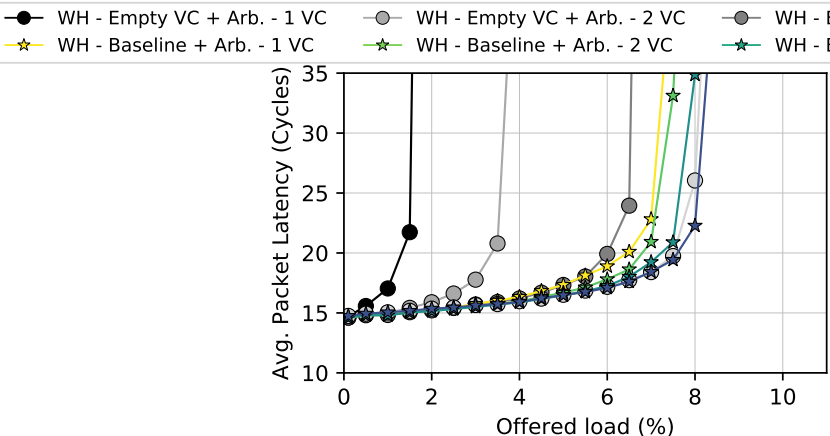

(a) DAMQ. Total size: 10 flits.

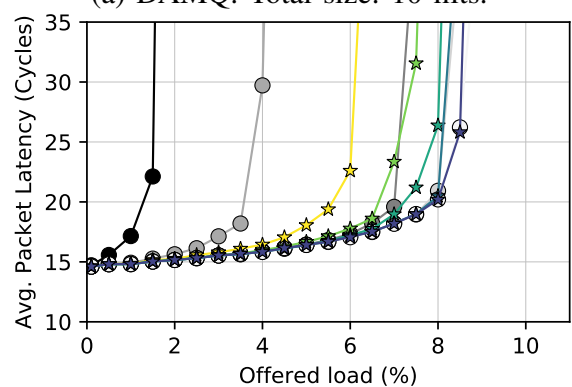

(c) Private VC. VC size: 5 flits.

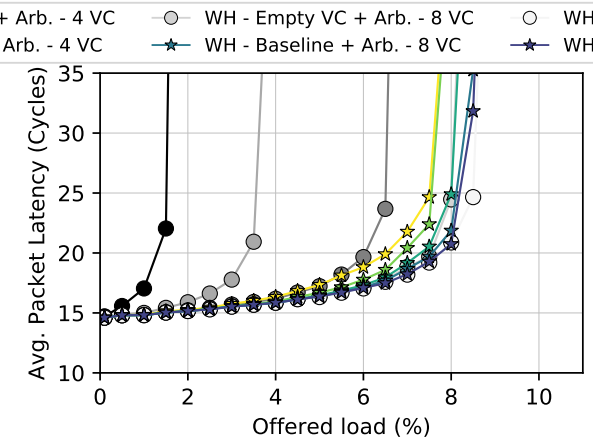

(b) DAMQ. Total size: 20 flits.

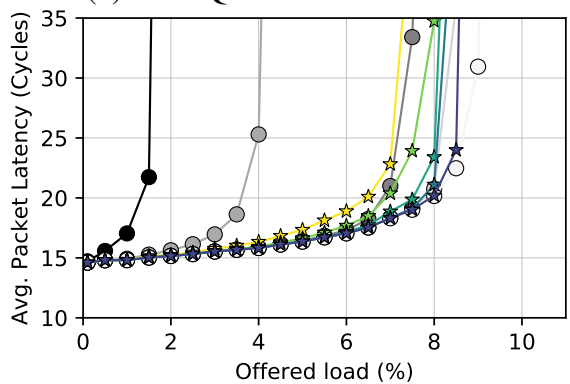

(d) Private VC. VC size: 10 flits.

Fig. 7: Packet latency in an $8 \times 8 c=4$ mesh with Empty $V C$ and WH flow controls, using bimodal traffic.

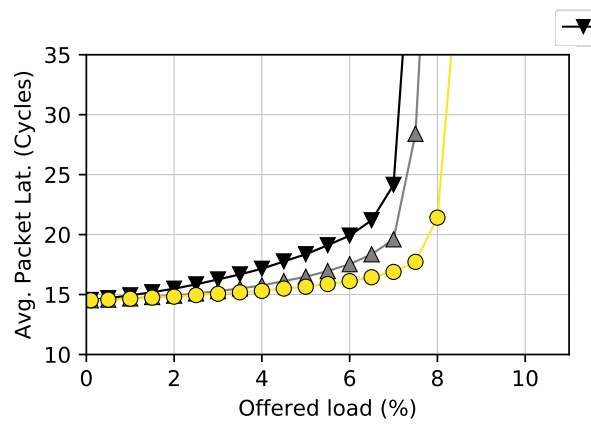

(a) Packet latency.

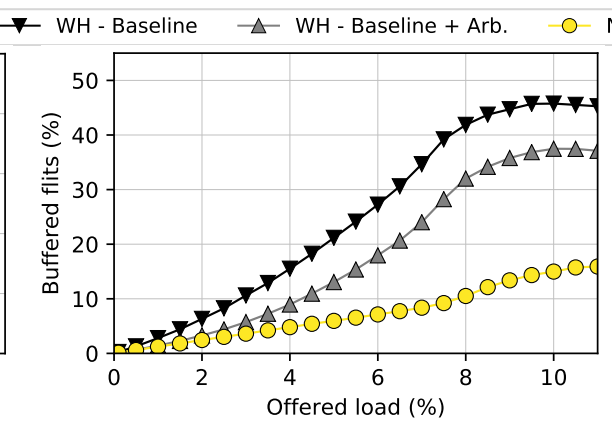

(b) Buffered flits.

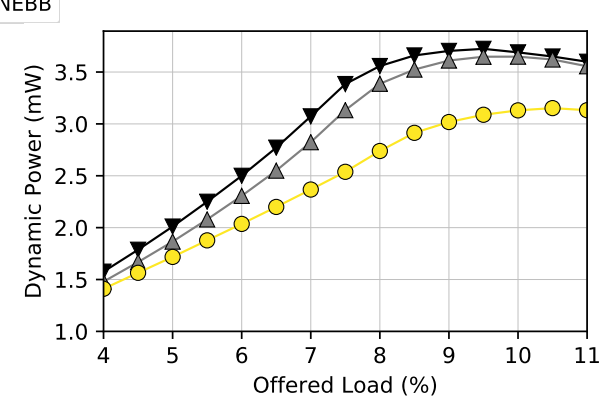

(c) Router dynamic power.

Fig. 8: $8 \times 8 c=4$ mesh performance and efficiency with uniform random single-flit traffic, a DAMQ of 6 flits and 2 VCs.

WH baseline. Next, the performance and efficiency of $N E B B$ using single-flit packets is considered; Bimodal traffic in mesh and torus is also evaluated. Finally, a sensitivity analysis is carried out.

\section{A. Empty VC vs WH Flow Control}

Figure 7 compares packet latency of two implementation of WH flow control, with the restriction of an empty buffer to forward data (Empty $V C$ ) or without it (WH-Baseline). Both versions have LA arbiters; this is the configuration with the best performance, as observed in the results presented in Section V-B. Bimodal traffic is used in all cases.

Figures $7 \mathrm{a}$ and $7 \mathrm{~b}$ employ shared buffers (DAMQ) with space for 10 and 20 flits, respectively. Clearly, the WH version has better results in respect of Empty $V C$ with 1, 2 and 4 VCs. It is particularly notorious the low throughput of Empty $V C$ with 1 and 2 VCs. WH-Baseline with a single VC is even better than Empty VC with 4 VCs. With 8 or more VCs, the improvement of WH-Baseline runs out, providing similar results in both configurations. Note that there are no results with $16 \mathrm{VCs}$ in Figure $7 \mathrm{a}$ because each VC needs a private slot and the DAMQ only has 10 flits.

Figures $7 \mathrm{c}$ and $7 \mathrm{~d}$ have a private buffer per $\mathrm{VC}$ with room for 5 and 10 flits, respectively. Again, WH-Baseline is better for 1 to $4 \mathrm{VCs}$, and similar with 8 and $16 \mathrm{VCs}$.

Reducing the amount of buffering space is critical to reduce power consumption and area in NoC designing. WH-Baseline is clearly better than Empty $V C$ with a low number of VCs and a low amount of buffering space. Therefore, we use this configuration as our baseline in the next sections.

\section{B. NEBB using Single-Flit Packets}

Figure 8 compares packet latency, buffered flits and dynamic power of each bypass mechanism; in all cases, lower values are better. These first evaluations use single-flit traffic, so all 


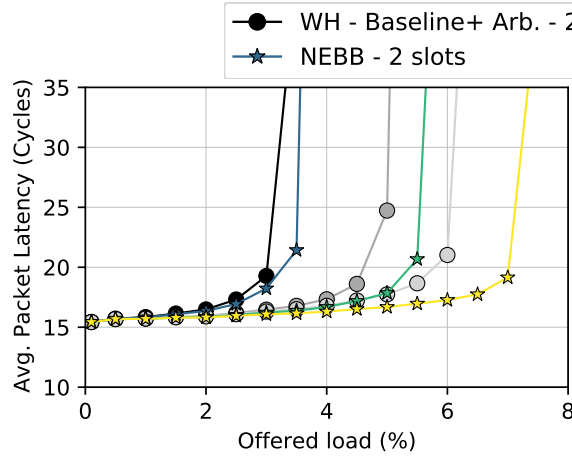

(a) Average packet latency.
O- WH - Baseline+ Arb. - 3 slots

N- NEBB - 3 slots

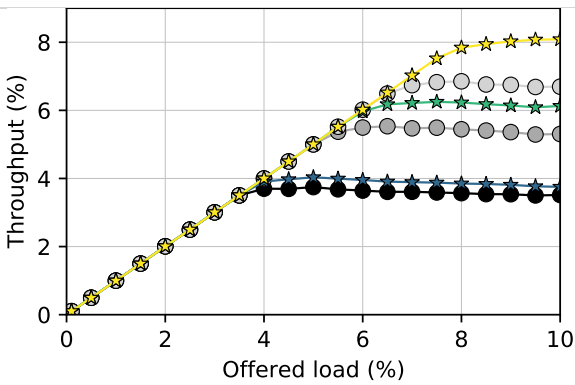

(b) Throughput.
O- WH - Baseline+ Arb. - 4 slots

NEBB - 4 slots

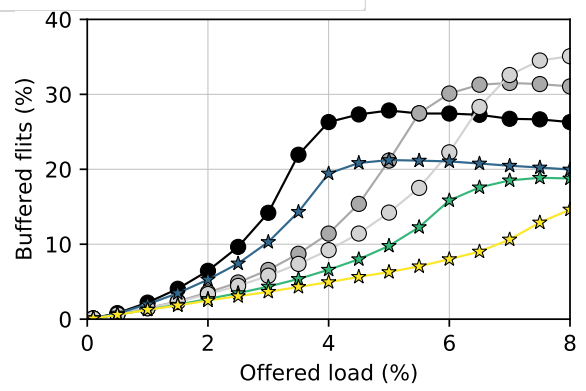

(c) Buffered flits.

Fig. 9: Performance of bypass routers in an $8 \times 8 c=4$ mesh with single-flit random-uniform traffic, an minimal buffering without VCs.

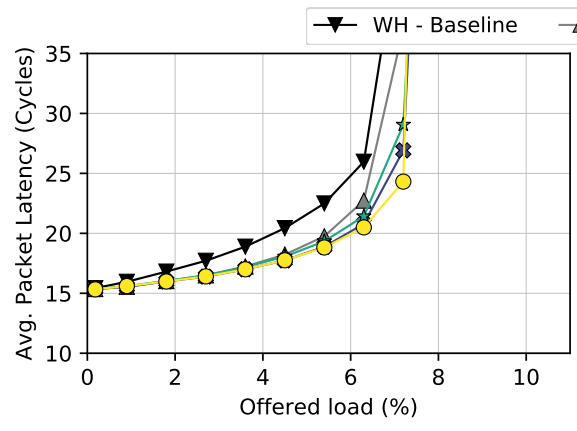

(a) Packet latency.

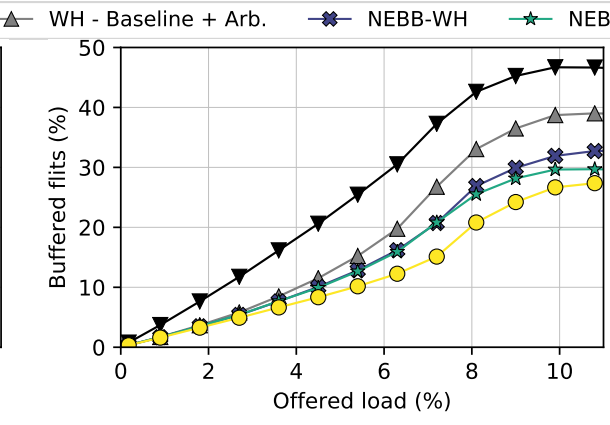

(b) Buffered flits.

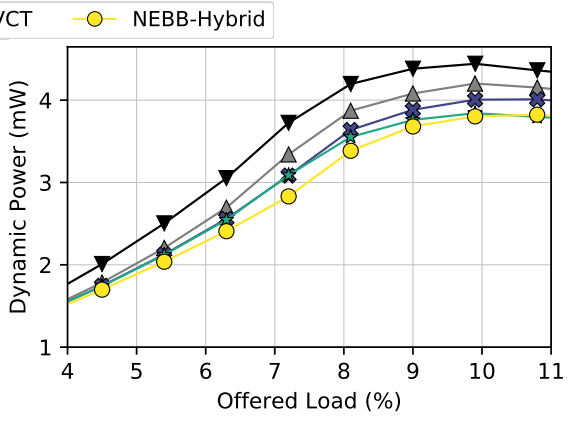

(c) Router dynamic power.

Fig. 10: $8 \times 8 c=4$ mesh performance and efficiency with uniform random and bimodal traffic, a DAMQ of 12 flits and 2 VCs.

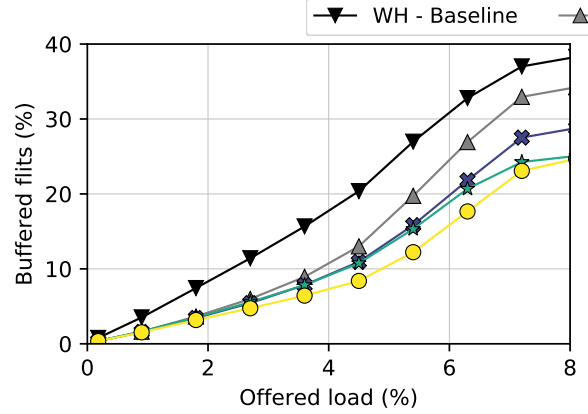

(a) Bit-reversal.

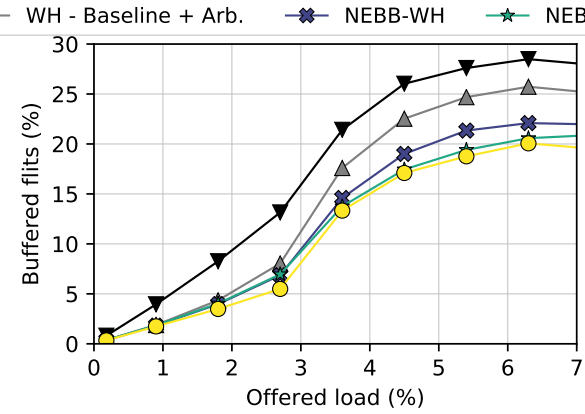

(b) Transpose.

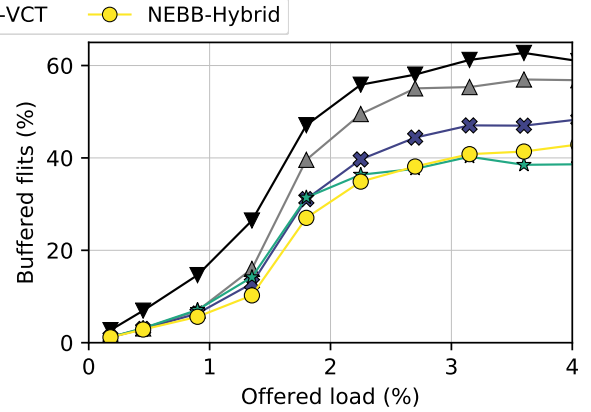

(c) Hotspot.

Fig. 11: Buffered flits in an $8 \times 8 c=4$ mesh for different traffic patterns, using bimodal traffic, a DAMQ of 12 flits and 2 VCs.

$N E B B$ variants are equivalent. Only 6 slots per shared buffer are used, adapted to the small packet size.

The amount of buffered flits in $8 \mathrm{~b}$ grows with the network load. WH-Baseline buffers flits when the buffers are non-empty or there are LA conflicts (all the conflicting LAs are discarded). The WH-Baseline $+A r b$ model is similar, but one LA proceeds in case of conflicts, reducing the use of buffers. In $N E B B$, buffers are only used when conflicts occur, not because of nonempty buffers, minimizing the buffer utilization. This translates into latency and power savings, particularly at intermediate loads. At $7 \%$ load (0.07 flits/node/cycle), $N E B B$ reduces $W H$ Baseline's latency in $30.1 \%$ and buffered flits in $75.9 \%$. From these values, $18.8 \%$ and $30.7 \%$ respectively come from the LA arbiter, as observed in $W H$-Baseline + Arb results. Regarding dynamic power, $N E B B$ saves $23.0 \%$ over $W H$-Baseline at $7 \%$ load.

Figure 9 shows the latency, throughput and buffered flits of $W H$-Baseline $+A r b$. and $N E B B$ using routers with minimal buffering. Evaluated configurations do not have VCs (i.e. equivalent to $1 \mathrm{VC}$ ) and the input buffers have 2,3 or 4 slots. Injected packets only have one flit; in this case, we do not plot results using 5-flit packets because NEBB-VCT cannot be applied. $N E B B$ reduces the number of buffered flits under these conditions by $24.5 \%, 39.3 \%$ and $51.3 \%$ for 2,3 , and 4 slots respectively. As a consequence of reducing Head of Line Blocking (HoLB) and the pressure over the buffers, 


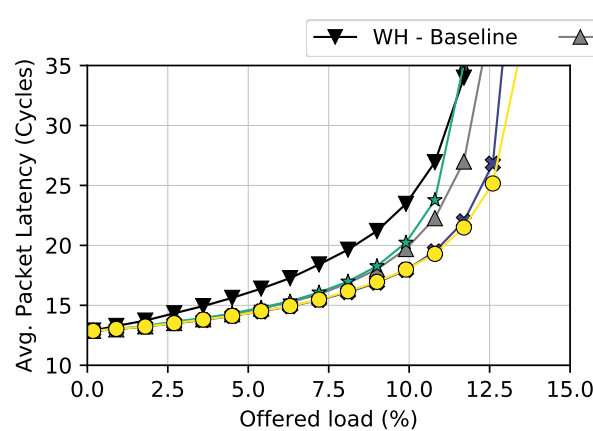

(a) Average packet latency.

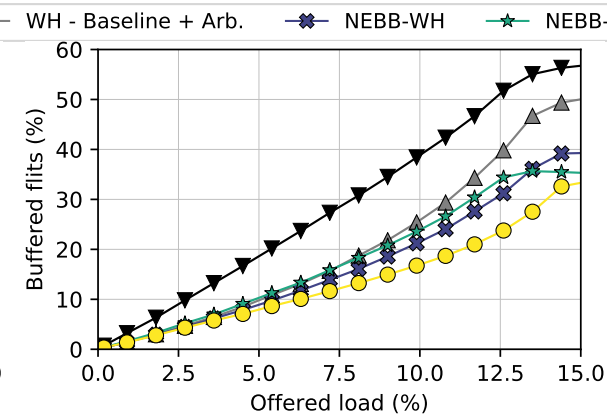

(b) Buffered flits.

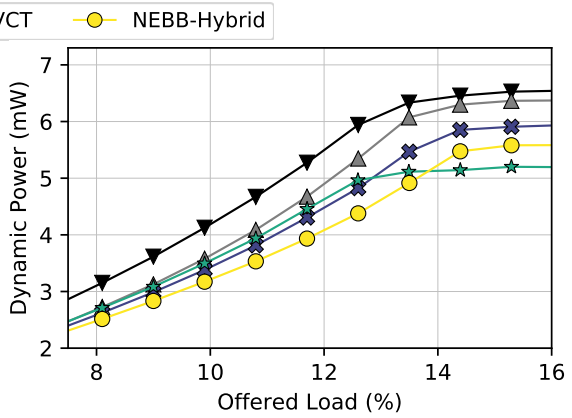

(c) Router dynamic power.

Fig. 12: Performance of an $8 \times 8 c=4$ torus with bimodal uniform traffic, a DAMQ of 12 flits and 2 VCs.

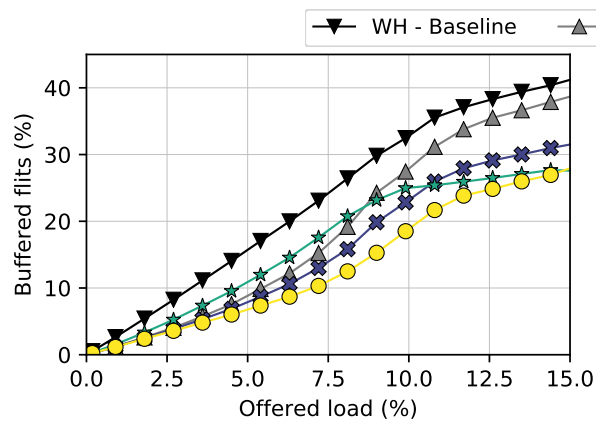

(a) Bit-reversal.

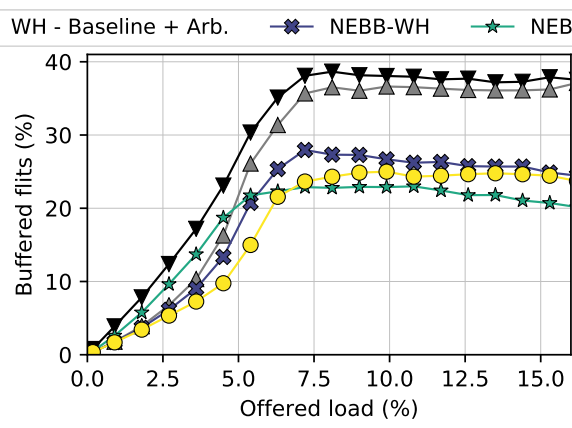

(b) Transpose.

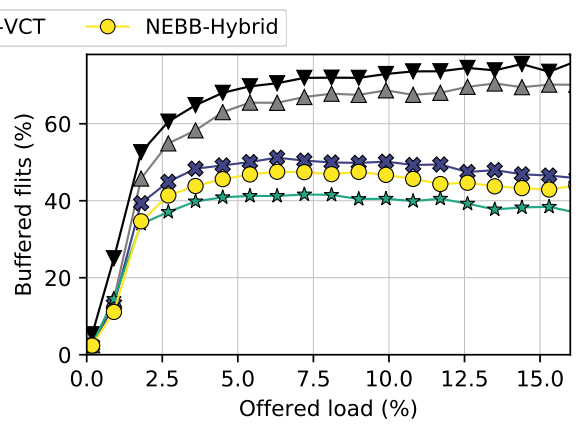

(c) Hotspot.

Fig. 13: Buffered flits in an $8 \times 8 c=4$ torus for different traffic patterns, using bimodal traffic, a DAMQ of 12 flits and 2 VCs.

$N E B B$ achieves $6.8 \%, 15.5 \%, 20.8 \%$ more throughput than WH-Baseline + Arb. for 2, 3 and 4 slots, respectively.

\section{NEBB Flow Control and Hybrid}

Figure 10 compares the $N E B B$ alternatives using bimodal traffic. The three $N E B B$ variants outperform the baselines, and Hybrid presents the best results since it maximizes the cases in which bypass is used.

Both $N E B B-W H$ and $N E B B-V C T$ present similar results. VCT has slightly higher latency and lower throughput, which translate into slightly lower power results after saturation.

NEBB-Hybrid has the best results in latency, buffered flits and dynamic power with a reduction of $20.6 \%, 60.1 \%$ and $21.1 \%$, respectively, over $\mathrm{WH}$-Baseline at a load around $6 \%$.

Figure 11 depicts the buffer utilization for different traffic patterns. The results are similar to the previous ones with uniform random traffic, with $N E B B$ mechanisms improving the utilization of the bypass and Hybrid being the optimal version.

\section{NEBB in Torus networks}

Figure 12 depicts results of the bypass router in a torus. All the bypass mechanisms use FBFC except for $N E B B-V C T$, which relies on Bubble flow control [28] since it only employs VCT.

In general, base latency is lower than in the mesh, and throughput is almost doubled, proving that FBFC and bypass routers with shared buffers operate correctly together. FBFC presents better results than Bubble, with $N E B B-V C T$ presenting worse latency than $W H$-Baseline $+A r b$. Once more, $N E B B$ Hybrid has the best results, improving latency over $W H$ Baseline by $28.4 \%$ and dynamic power by $24.4 \%$ at $11 \%$ load.

Figure 13 shows the buffer utilization applying different traffic patterns. $N E B B$ always writes less flits in the buffers than the baselines. In general, NEBB-Hybrid is the best choice between the $N E B B$ mechanisms, except in the case of the hotspot pattern, in which $N E B B-V C T$ is slightly better.

\section{E. Real traffic analysis}

This section evaluates $N E B B$-Hybrid with real traffic through FS simulations in meshes and tori. We compare $N E B B$ Hybrid with WH-Empty $V C+A r b$ and $W H$-Baseline $+A r b$. All configurations have shared buffers of 12 flits with $3 \mathrm{VCs}, 1$ VC per VN to avoid protocol-deadlock, except for Torus-WHEmpty $V C$ that has $6 \mathrm{VCs}$ of 5 slots each. The reason is that it does not support FBFCL, so it it uses Dateline to avoid routing-deadlock, requiring $2 \mathrm{VCs}$ per $\mathrm{VN}$.

Figure 14 shows packet latency and offered load. First, the offered load in these benchmarks is very low, around $2 \%$ in canneal, the benchmark with the highest load. For this reasons the differences between WH-Baseline + Arb and NEBB-Hybrid are small as we have shown in the previous sections with synthetic traffic. Second, the configurations that use a torus reduce latency with respect to their mesh counterpart. Third, the WH-Empty $V C+A r b$ configurations have on average $44.9 \%$ and $27.3 \%$ more latency in the mesh and torus, respectively, compared with WH-Baseline+Arb. This is because WH-Empty 


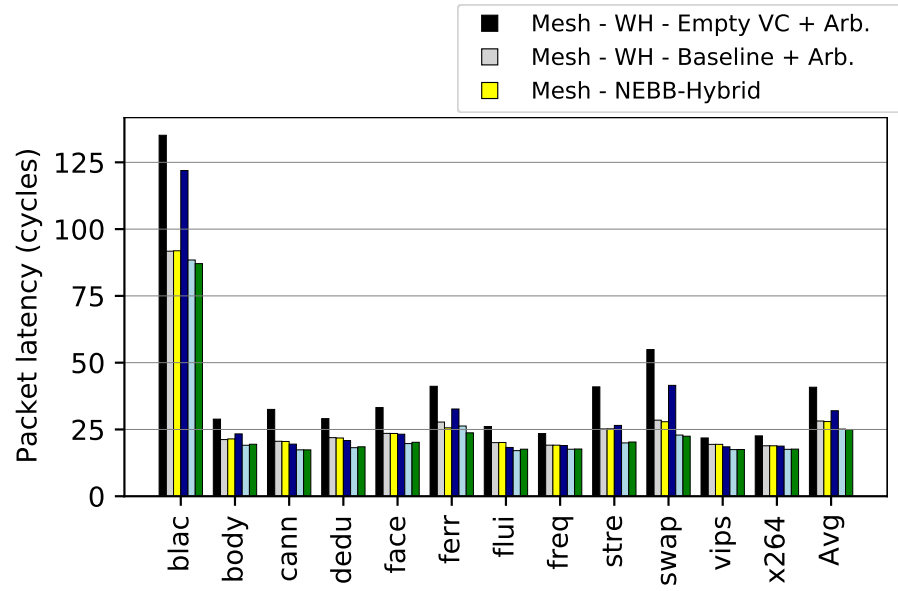

(a) Avg. packet latency

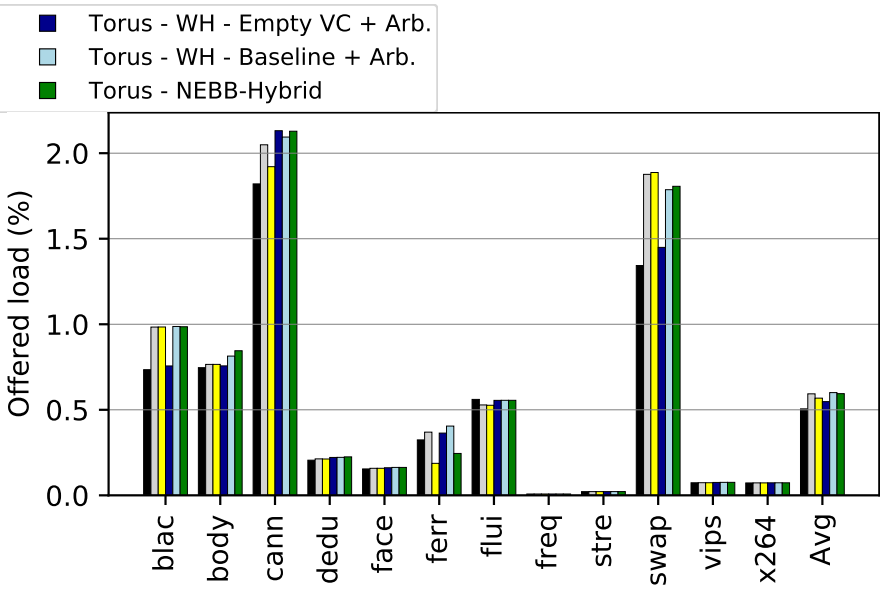

(b) Offered load

Fig. 14: Real-traffic performance.

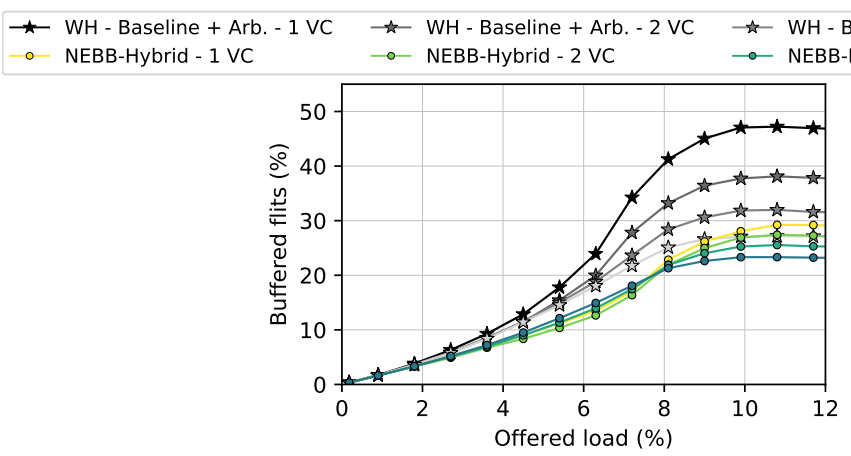

(a) DAMQ. Total size: 10 flits.

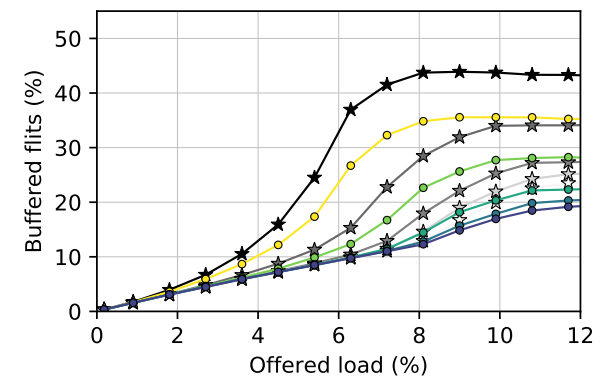

(c) Private VC. VC size: 5 flits.

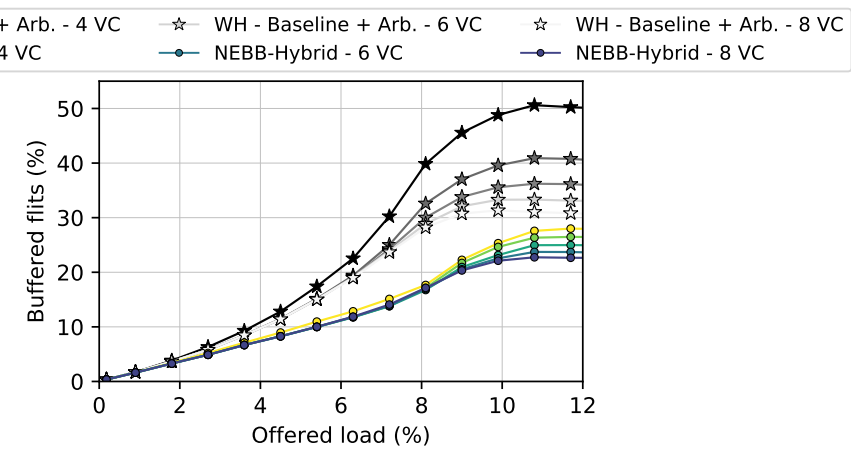

(b) DAMQ. Total size: 20 flits.

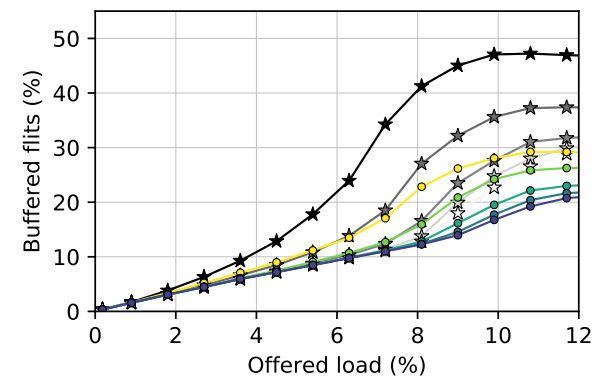

(d) Private VC. VC size: 10 flits.

Fig. 15: Buffer utilization for a mesh with different number of VCs and buffer sizes using bimodal traffic.

$V C+A r b$ requires more $\mathrm{VCs}$ to offer the same performance as shown in Section V-A. In the case of the torus, it is remarkable that the FBFCL configurations get lower latency with half the VCs and require less overall buffer space due to Dateline's inefficient VCs utilization.

\section{F. Sensitivity analysis: buffer depth, starvation}

1) Buffer depth and number of VCs: Figure 15 depicts the buffer utilization for WH-Baseline+Arb and Hybrid with different combinations of VCs and buffer sizes. Each curve represents the same configuration with a different number of VCs, either sharing the same buffer space (15a and $15 \mathrm{~b}$ ) or using private buffers per VC (15c and $15 \mathrm{~d})$. With shared buffers, Hybrid clearly outperforms WH-Baseline $+A r b$, particularly when the shared buffer size is not very small. With 20 flits per port, no amount of VCs in WH-Baseline +Arb matches the result of Hybrid. The amount of VCs used in Hybrid has a small impact on buffered flits.

In the private buffers evaluations in $15 \mathrm{c}$ and $15 \mathrm{~d}$ the total amount of storage increases with the $\mathrm{VC}$ count. If buffers are very small (15c, buffer per VC equals the maximum packet size of 5 flits) Hybrid is better than WH-Baseline+Arb for the same number of VCs, but the improvement is modest. Indeed, this is the minimum buffer size for Hybrid to use VCT. With larger buffers in $15 \mathrm{~d}$, the results of Hybrid with half the VCs approximately match the result of WH-Baseline + Arb before saturation, and get better after this point. 


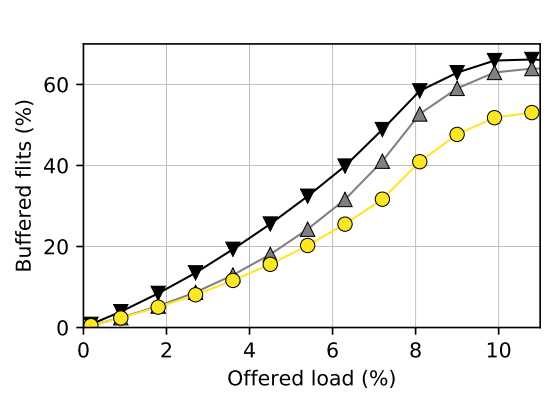

(a) Buffered flits. Priority to flits.

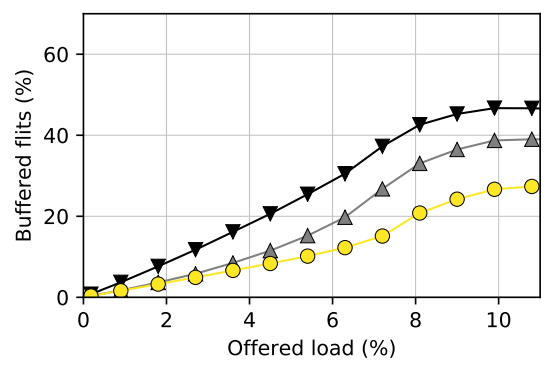

(d) Buffered flits. Priority to LAs.

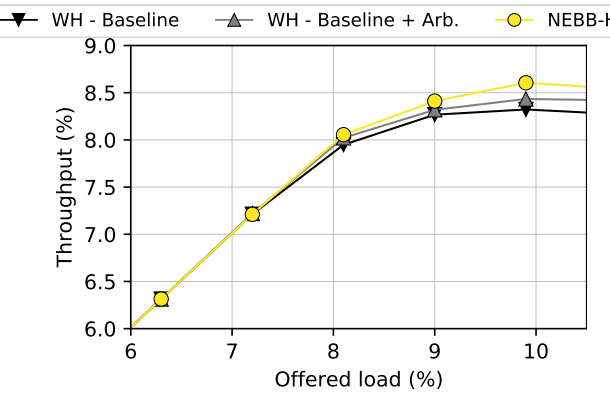

(b) Throughput. Priority to flits.

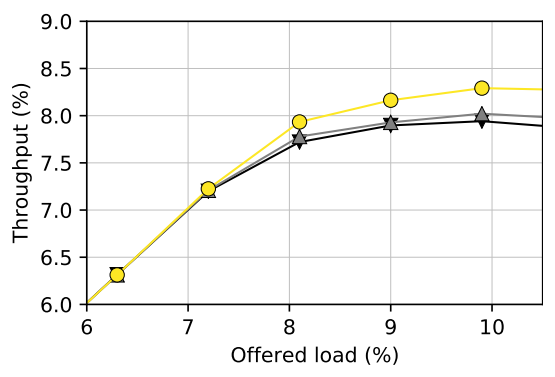

(e) Throughput. Priority to LAs.

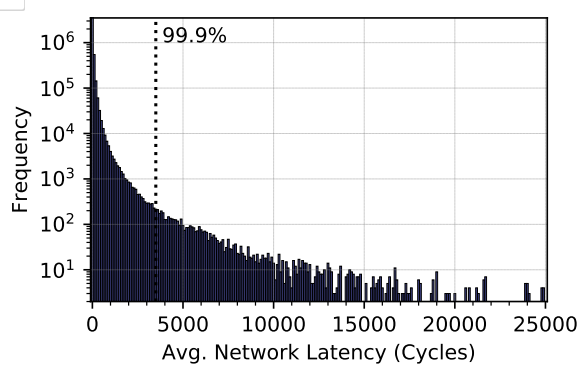

(c) Latency histogram in saturation (load 10\%). Priority to flits.

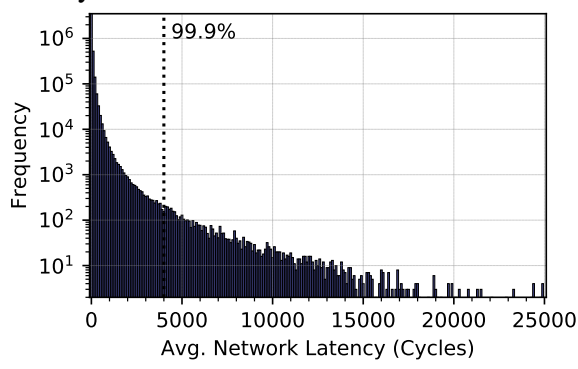

(f) Latency histogram in saturation (load 10\%). Priority to LAs.

Fig. 16: NEBB-Hybrid buffer utilization, throughput and network latency histograms prioritizing buffered flits or LAs in case of conflicts.

2) Crossbar priority to buffered or bypassed flits: The original bypass condition 2 in Section II-B prioritizes flits in the non-bypass pipeline, but the opposite priority (to LAs) is used in this paper. Figure 16 compares both alternatives, depicting buffered flits and packet latency histograms. On the one hand, giving priority to LAs decreases significantly the number of buffered flits in all mechanisms, particularly at medium and high load. On the other hand, the maximum throughput decreases slightly.

The packet latency histograms of Hybrid show that the number of high latency packets increases with priority to LAs. This issue is shared by all mechanisms when more than $1 \mathrm{VC}$ is used. To reduce peak latency, priority may be given to buffered flits after a given number of cycles. The specific threshold used (e.g. 30 cycles in [3]) presents a tradeoff between the results with priority to LAs or to buffered flits.

\section{RELATED WORK}

Sections I and II have already presented LookAhead [1] and bypass [2] mechanisms. Token Flow Control (TFC, [3]) communicates information about the availability of resources among nodes in a neighborhood. The objective of the mechanism is the improvement of the bypass utilization by choosing low congested paths, exploiting path diversity with adaptive routing.

Other works attack the ordering issues in NOCs. Scorpio [29] implements a globally ordered mesh for snoopy coherence protocols that addresses the storage scalability problem of directory-based protocols. In general terms, it solves the global message order required by snoopy protocols (which is not intrinsic to mesh topologies) with a broadcast network, which is used to inform the Network Interface Controllers (NICs) about the correct order of the messages. When the protocol messages are received in the NICs, they are reorganized according to the previous information. Since the micro-architecture of the routers used in Scorpio is similar to the one describe in Section II, we presume that it can be combined with NEBB to enhance performance.

Our Hybrid approach combines two flow controls, WH and VCT. Whole Packet Forwarding (WPF, [25]) applies packet-based flow control in a WH network, but they do it to relax VC re-allocation requirements in deadlock-free fully adaptive routing NoCs, without considering bypass. In [30] they suggest using two different types of flow control, buffered and unbuffered, but again without considering bypass.

The mechanism in SMART [31], implemented in the OpenSMART NoC generator [32], extends bypass mechanisms to skip multiple routers in a single cycle, multiplying the savings in latency and power. The bypass conditions need to be satisfied in all the routers in the path. For this reason, we believe that our Hybrid bypass mechanism can be applied to such designs. A detailed analysis of multi-hop bypass mechanisms based on $N E B B$ is left for future work. Preliminary results have been reported in [33] with promising improvements in terms of the number of VCs and the required buffer size.

ShortPath [34] proposes an alternative pipeline organization for bypass routers that enhances performance by avoiding the speculative allocation of this kind of routers. Its architecture minimizes the time spent by each flit in a router by allowing the bypass of part of the allocation pipeline stages, i.e. only the first stage even when the LA loses in the second stage. As far as we know, ShortPath uses WH (i.e. flit level flow 
control) as it performs SA for body flits, and allows the storage of multiple packets in the same input VC buffer. Combining ShortPath with NEBB is an interesting idea for future work that may increase the utilization of the bypass paths.

\section{CONCLUSIONS}

Bypass reduces packet latency and power consumption, which are key aspects of NoC designs. Our Non-Empty Buffer Bypass proposal is based on a proper analysis and relaxing of the original bypass conditions. Variants of $N E B B$ following $\mathrm{WH}$ and VCT rules are introduced, and the combination of them, denoted Hybrid, maximizes the utilization of the bypass.

We show the effectiveness of $N E B B$ and Hybrid in a mesh and a torus using FBFC. Our proposals decrease by up to $30 \%$ packet latency and up to $23 \%$ dynamic power savings in relation to applying WH flow control. Additionally, results show that Hybrid outperforms prior proposals with shared buffers, and requires half the VCs for the same result with private buffers, what simplifies VC allocation.

Altogether, these results present Hybrid as a competitive and cost-effective alternative to improve the design and performance of the NoC bypass techniques in NoC routers.

\section{REFERENCES}

[1] M. Galles, "Spider: a high-speed network interconnect," IEEE Micro, vol. 17, pp. 34-39, Jan 1997.

[2] A. Kumar, P. Kunduz, A. Singhx, L.-S. Pehy, and N. Jhay, "A 4.6 Tbits/s 3.6 GHz single-cycle NoC router with a novel switch allocator in $65 \mathrm{~nm}$ CMOS," in International Conference on Computer Design (ICCD), 2007.

[3] A. Kumar, L.-S. Peh, and N. K. Jha, "Token flow control," in International Symposium on Microarchitecture (Micro), pp. 342-353, 2008.

[4] P. Gratz, B. Grot, and S. W. Keckler, "Regional congestion awareness for load balance in networks-on-chip," in International Symposium on High Performance Computer Architecture (HPCA), Feb 2008.

[5] B. Fu, Y. Han, J. Ma, H. Li, and X. Li, "An abacus turn model for time/space-efficient reconfigurable routing," in International Symposium on Computer Architecture (ISCA), 2011.

[6] E. Nilsson, M. Millberg, J. Oberg, and A. Jantsch, "Load distribution with the proximity congestion awareness in a network on chip," in Design Automation and Test in Europe, pp. 1126-1127, 2003.

[7] T. Moscibroda and O. Mutlu, "A case for bufferless routing in on-chip networks," in International Symposium on Computer Architecture, 2009.

[8] R. Ausavarungnirun, C. Fallin, X. Yu, K. K.-W. Chang, G. Nazario, R. Das, G. H. Loh, and O. Mutlu, "A case for hierarchical rings with deflection routing," Parallel Comput., vol. 54, pp. 29-45, May 2016.

[9] C. A. Nicopoulos, D. Park, J. Kim, N. Vijaykrishnan, M. S. Yousif, and C. R. Das, "ViChaR: A dynamic virtual channel regulator for networkon-chip routers," in International Symposium on Microarchitecture, 2006

[10] I. Perez, E. Vallejo, and R. Beivide, "Efficient router bypass via hybrid flow control," in 2018 11th International Workshop on Network on Chip Architectures (NoCArc), pp. 1-6, IEEE, 2018.

[11] S. Ma, Z. Wang, Z. Liu, and N. E. Jerger, "Leaving one slot empty: Flit bubble flow control for torus cache-coherent NoCs," IEEE Transactions on Computers, vol. 64, no. 3, pp. 763-777, 2015.

[12] T. Krishna, J. Postman, C. Edmonds, L.-S. Peh, and P. Chiang, "SWIFT: A SWing-reduced Interconnect For a Token-based network-on-chip in 90nm CMOS," in International Conference on Computer Design, 2010.

[13] C. Bienia, S. Kumar, J. P. Singh, and K. Li, "The parsec benchmark suite: Characterization and architectural implications," in Proceedings of the 17th international conference on Parallel architectures and compilation techniques, pp. 72-81, 2008.

[14] N. E. Jerger, T. Krishna, and L.-S. Peh, On-Chip Networks, Second Edition, vol. 12. 2017.

[15] D. U. Becker, N. Jiang, G. Michelogiannakis, and W. J. Dally, "Adaptive backpressure: Efficient buffer management for on-chip networks," in International Conference on Computer Design (ICCD), 2012.

[16] A. Sodani, "Knights landing (KNL): 2nd generation Intel® Xeon Phi processor," in Hot Chips 27 Symposium (HCS), pp. 1-24, 2015.
[17] A. Kumar, "The new Intel Xeon processor scalable family (formerly Skylake-SP)," in Hot Chips 29 Symposium (HCS), 2017.

[18] S. Davidson, S. Xie, C. Torng, K. Al-Hawai, A. Rovinski, T. Ajayi, L. Vega, C. Zhao, R. Zhao, S. Dai, A. Amarnath, B. Veluri, P. Gao, A. Rao, G. Liu, R. K. Gupta, Z. Zhang, R. Dreslinski, C. Batten, and M. B. Taylor, "The Celerity open-source 511-core RISC-V tiered accelerator fabric: Fast architectures and design methodologies for fast chips," IEEE Micro, vol. 38, pp. 30-41, Mar 2018.

[19] W. Dally and B. Towles, Principles and Practices of Interconnection Networks. San Francisco, CA, USA: Morgan Kaufmann Inc., 2003.

[20] V. Puente, C. Izu, R. Beivide, J. A. Gregorio, F. Vallejo, and J. M. Prellezo, "The adaptive bubble router," Journal of Parallel and Distributed Computing, vol. 61, no. 9, pp. 1180-1208, 2001.

[21] Y. Tamir and G. L. Frazier, "Dynamically-allocated multi-queue buffers for vlsi communication switches," IEEE Transactions on Computers, vol. 41, pp. 725-737, Jun 1992.

[22] M. Shin and J. Kim, "Leveraging torus topology with deadlock recovery for cost-efficient on-chip network," in 2011 IEEE 29th International Conference on Computer Design (ICCD), pp. 25-30, 2011.

[23] A. Singh, W. J. Dally, A. K. Gupta, and B. Towles, "Goal: a loadbalanced adaptive routing algorithm for torus networks," in 30th Annual International Symposium on Computer Architecture, 2003. Proceedings., pp. 194-205, IEEE, 2003.

[24] N. Jiang, J. Balfour, D. U. Becker, B. Towles, W. J. Dally, G. Michelogiannakis, and J. Kim, "A detailed and flexible cycle-accurate networkon-chip simulator," in International Symposium on Performance Analysis of Systems and Software (ISPASS), pp. 86-96, 2013.

[25] S. Ma, N. E. Jerger, and Z. Wang, "Whole Packet Forwarding: Efficient design of fully adaptive routing algorithms for networks-on-chip," in High-Performance Computer Architecture (HPCA), 2012.

[26] N. Binkert, B. Beckmann, G. Black, S. K. Reinhardt, A. Saidi, A. Basu, J. Hestness, D. R. Hower, T. Krishna, S. Sardashti, et al., "The gem5 simulator," ACM SIGARCH computer architecture news, vol. 39, no. 2, pp. $1-7,2011$

[27] C. Sun, C.-H. O. Chen, G. Kurian, L. Wei, J. Miller, A. Agarwal, L.-S. Peh, and V. Stojanovic, "DSENT-a tool connecting emerging photonics with electronics for opto-electronic networks-on-chip modeling," in International Symposium on Networks on Chip (NoCS), 2012.

[28] C. Carrion, R. Beivide, J. A. Gregorio, and F. Vallejo, "A flow control mechanism to avoid message deadlock in k-ary n-cube networks," in International Conference on High-Performance Computing, 1997.

[29] B. K. Daya, C.-H. O. Chen, S. Subramanian, W.-C. Kwon, S. Park, T. Krishna, J. Holt, A. P. Chandrakasan, and L.-S. Peh, "Scorpio: a 36core research chip demonstrating snoopy coherence on a scalable mesh noc with in-network ordering," in 2014 ACM/IEEE 41st International Symposium on Computer Architecture (ISCA), pp. 25-36, IEEE, 2014.

[30] S. A. R. Jafri, Y. J. Hong, M. Thottethodi, and T. N. Vijaykumar, "Adaptive flow control for robust performance and energy," in International Symposium on Microarchitecture (Micro), pp. 433-444, 2010.

[31] T. Krishna, C.-H. O. Chen, W. C. Kwon, and L.-S. Peh, "Breaking the on-chip latency barrier using smart," in 2013 IEEE 19th International Symposium on High Performance Computer Architecture (HPCA), pp. 378-389, IEEE, 2013.

[32] H. Kwon and T. Krishna, "OpenSMART: Single-cycle multi-hop NoC generator in BSV and Chisel," in International Symposium on Performance Analysis of Systems and Software (ISPASS), pp. 195-204, 2017.

[33] I. Pérez, E. Vallejo, and R. Beivide, "SMART++ reducing cost and improving efficiency of multi-hop bypass in NoC routers," in Proceedings of the 13th IEEE/ACM International Symposium on Networks-on-Chip, pp. 1-8, 2019.

[34] A. Psarras, I. Seitanidis, C. Nicopoulos, and G. Dimitrakopoulos, "Shortpath: A network-on-chip router with fine-grained pipeline bypassing," IEEE Trans. Comput. (TC), vol. 65, pp. 3136-3147, Oct 2016. 


\section{BIOGRAPHY}

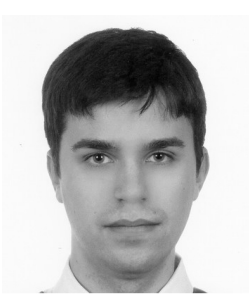

Iván Pérez is a PhD student in Computer Architecture in the University of Cantabria, Spain. He received the B. Sc. degree in Telecomunication Engineer form the University of Cantabria in 2014. His research interests include networks on chip and memory hierarchy of manycore processors.

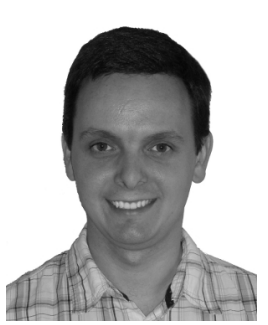

Enrique Vallejo received the $\mathrm{PhD}$ degree in computer architecture from the University of Cantabria, in 2010. He is an assistant professor with the University of Cantabria, where he lectures Interconnection Networks in the Computer Science studies. His research interests cover different areas of parallel computing: Interconnection networks, processor microarchitecture, transactional memory, and synchronization mechanisms.

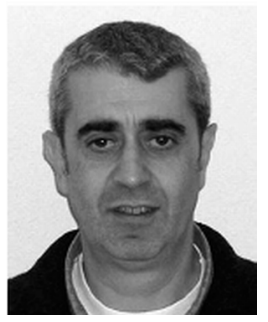

Ramón Beivide received a $\mathrm{PhD}$ degree in computer science and engineering from the Universidad Politecnica de Catalunya (UPC), Barcelona, in 1985. $\mathrm{He}$ has been an associate professor at UPC and the Universidad del Pais Vasco. In 1991, he joined the Universidad de Cantabria in Santander, Spain, where he is a full professor of telecommunication and computer engineering and he has served as the dean of the School of Computer Science. His research interests include parallel computers, interconnection networks, memory hierarchies and graph theory. $\mathrm{He}$ has published more than 100 technical papers on such topics. 